\title{
Correction to: Multigene panel testing for hereditary breast and ovarian cancer in the province of Ontario
}

\author{
Jordan Lerner-Ellis ${ }^{1,2,3}$ - Chloe Mighton ${ }^{2,4,5} \cdot$ Conxi Lazaro $^{2,6,7} \cdot$ Nicholas Watkins $^{2,8}$. Vanessa Di Gioacchino ${ }^{2}$. \\ Andrew Wong ${ }^{2} \cdot$ Martin C. Chang ${ }^{1,9}$. George S. Charames ${ }^{1,2,3}$
}

Published online: 25 September 2020

(c) Springer-Verlag GmbH Germany, part of Springer Nature 2020

\section{Correction to: \\ Journal of Cancer Research and Clinical Oncology https://doi.org/10.1007/s00432-020-03377-6}

In the original article published, the proportions for the categories "Non-AJ, non-FC, non-Hispanic White" and "Other ethnicity" have incorrectly been reversed. The correct values for "Non-AJ, non-FC, non-Hispanic White" should be 62.3\% (2024) and "Other ethnicity" should be $4.2 \%$ (136).

Publisher's Note Springer Nature remains neutral with regard to jurisdictional claims in published maps and institutional affiliations.

The original article can be found online at https://doi.org/10.1007/ s00432-020-03377-6.

Jordan Lerner-Ellis

Jordan.Lerner-Ellis@SinaiHealth.ca

1 Department of Laboratory Medicine and Pathobiology, University of Toronto, 600 University Avenue, Toronto, ON M5G 1X5, Canada

2 Department of Pathology and Laboratory Medicine, Mount Sinai Hospital, Sinai Health, Toronto, ON, Canada

3 Lunenfeld-Tanenbaum Research Institute, Sinai Health, Toronto, ON, Canada

4 Institute of Health Policy, Management and Evaluation, University of Toronto, Toronto, ON, Canada
5 Genomics Health Services Research Program, Li Ka Shing Knowledge Institute, St. Michael's Hospital, Toronto, ON, Canada

6 Hereditary Cancer Program, ICO-IDIBELL, Barcelona, Spain

7 Women's College Research Institute, Women's College Hospital, Toronto, ON, Canada

8 Department of Molecular Genetics, University of Toronto, Toronto, ON, Canada

9 University of Vermont Cancer Center, Burlington, VT, USA 